\title{
Algoritmos ART para a Estimativa do Coeficiente de Absorção com Feixes Divergentes usando certas Funções de Bregman
}

M.J. BERROCAL TITO ${ }^{1}$, R.F. CARITA MONTERO ${ }^{2}$, Instituto de Matemática Pura e Aplicada (IMPA), Est. D. Castorina 110, 22460-320 Rio de Janeiro, RJ, Brasil.

Resumo. Consideramos uma classe de algoritmos tipo ART (Algebraic Reconstruction Technique) empregando certas funções de Bregman, para a estimativa do coeficiente de absorção com feixes divergentes de poucas vistas onde o número de incógnitas é maior ao número de dados. O funcional de Bregman usado está relacionado às entropias estudadas por Shannon, Harvda-Charvát e Sharma-Taneja. Este funcional depende de certos parâmetros $r$ e $s$. Com este funcional nós construímos uma família de distâncias de Bregman para ser empregada nos algoritmos ART. Neste trabalho procuram-se os valores ótimos dos parâmetros $r$ quando $s \rightarrow 1$, que ofereçam os melhores resultados para a estimativa dos coeficientes de absorção. Casos testes são apresentados, empregando dados com ruídos randômicos.

\section{Introdução}

A tomografia de raios $\mathrm{X}$ representa talvez o problema inverso mais básico onde temos que determinar a estrutura interna de um meio baseado em dados obtido externamente por detectores.

Problemas relativamente simples são os ensaios não destrutivos com aplicação tecnológica importante na indústria, diagnose e tratamento em medicina, e no qual o espalhamento pode ser desprezado, sendo a absorção (denominado atenuação em medicina) o modo dominante de interação da radiação no meio. A Tomografia computadorizada (CT) e a tomografia computadorizada por emissão de fótons (SPECT) estão dentro deste contexto $[6,3,5]$.

Uma aproximação muito boa é considerar que os raios $\mathrm{X}$ viajam por qualquer meio em linha reta. Para medir os raios depois que eles atravessarem uma região de interesse, o detector é colocado diretamente em frente a uma fonte de raios X, como mostrado na Figura 1. Assume-se que os raios X não se espalham e que serem apenas parcialmente absorvidos pelo meio. Assim a intensidade, $I$, que atravessa uma diferencial do meio, $d s$, pode ser descrita por

\footnotetext{
1 mabet99@yahoo.com

${ }^{2}$ rfcm20000@yahoo.com.br
} 


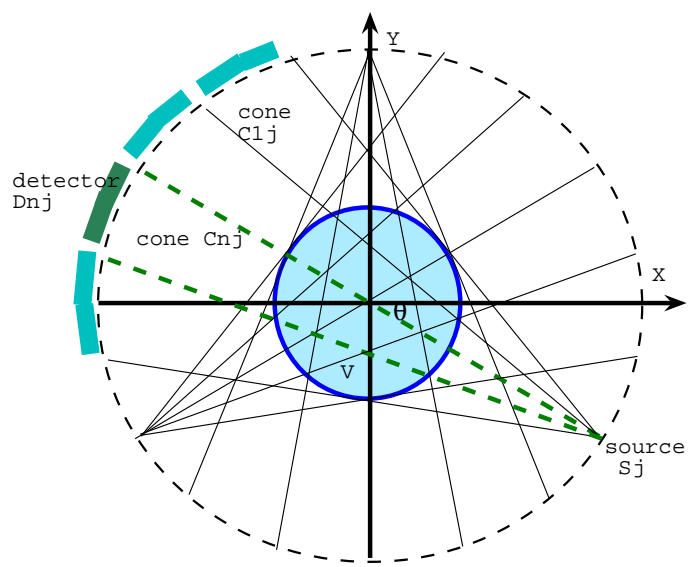

Figura 1: Geometria básica e partição do domínio com feixes divergentes.

$$
\frac{d I}{I}=-f(x, y) d s
$$

onde a função $f(x, y)$ esta relacionada ao coeficiente de atenuação, $\sigma$. Assumindo uma intensidade $I_{S}$ na fonte, a Eq.(1.1) pode ser integrada dada a intensidade do detector $I_{D}$ como

$$
I_{D}=I_{S} \exp \left\{\int_{\text {cone }} f(x, y) d s\right\} \Rightarrow \ln \frac{I_{S}}{I_{D}}=\int_{\text {cone }} f(x, y) d s .
$$

Em tomografia de raio $\mathrm{X}$, o problema é recuperar $f(x, y)$ desde sua integral ao longo do caminho entre a fonte - detector. Neste trabalho consideramos cones como os caminhos onde a radiação passa. Na Fig. 1, uma linha de detectores é organizada em frente de uma fonte. Os dados são colecionados em função do ângulo de giro, $\theta$, feito pela linha fonte e o eixo $x$. O sistema é girado ao redor do objeto, cada giro do sistema denomina-se vista $j, j=1,2, \ldots, J$. A integral da Eq.(1.2) tem a forma $[2]$

$$
\int_{\text {cone }} f(x, y) d s=\sum_{e \in c_{j, n_{j}}}^{E} \sigma_{e} a_{e}, \quad e=1,2, \ldots, E \quad e \quad n_{j}=1,2, \ldots, N_{j},
$$

onde $n_{j}$, indica um cone, caminho relacionado com a posição entre a fonte e o detector, $n_{j}=1,2, \ldots, N_{j} . N_{j}$ é o número total de cones do sistema $j, j=1,2, \ldots, J$, $j$ é um sistema rotado por um ângulo $\theta, J$ o número total de sistemas rotados, $a_{e}$ é um elemento de área do domínio discretizado, $e=1,2, \ldots, E$, onde $E$ é o número total de elementos de área que discretizam o domínio.

\section{Partição do Domínio}

A partição do domínio consistente com o sistema fonte-detector representado na Figura 1. consiste na identificação e numeração dos polígonos formados pela in- 
terseção de todos os cones originadas com todas as fontes bem como a identificação a numeração e a ordenação dos vértices destes polígonos. Esta partição assim gerada é chamada de base natural. Uma descrição detalhada sobre a partição do domínio com feixes divergentes é encontrada em $[2,7]$.

\section{Funções Convexas e Entropias}

Seja $z=\left\{z_{1}, z_{2}, \ldots, z_{p}\right\}$ o vetor formado pelas incógnitas positivas de um sistema. Define-se o seguinte funcional

$$
\eta_{s, r}\left(z_{i}\right)=\frac{z_{i}^{s}-z_{i}^{r}}{s-r} \quad c o m \quad s>1, \quad r \in(0,1] \quad \text { or } \quad r>1, \quad s \in(0,1]
$$

que está relacionada com a entropia de Sharma e Taneja [9], Shannon [8] e de Havrda - Charvát [4]

- se $s \rightarrow 1$ e $r \rightarrow 1$ obtemos $\eta_{1,1}\left(z_{i}\right)=z_{1} \ln z_{i}$. (entropia de Shannon)

- se $s \rightarrow 1$ e $r>0$ obtemos $\eta_{1, r}\left(z_{i}\right)=\left(z_{i}-z_{i}^{r}\right) /(1-r)$. (entropia de HavrdaCharvát).

A distância de Bregman [1] é definida como

$$
D\left(z, z_{0}\right)=D\left(\eta(z), \eta\left(z_{0}\right)\right)=\eta(z)-\eta\left(z_{0}\right)-\left\langle\nabla \eta\left(z_{0}\right), z-z_{0}\right\rangle
$$

onde $\nabla$ é um operador gradiente

$$
\left\langle\nabla \eta\left(z_{0}\right), z-z_{0}\right\rangle=\left.\frac{\partial \eta}{\partial z}\right|_{z_{0}}\left(z-z_{0}\right)
$$

Com o funcional $\eta_{s, r}$ obtemos as seguintes distâncias de Bregman.

$$
D_{s, r}\left(z, z_{0}\right)=\frac{1}{s-r}\left[\sum_{i=1}^{p}\left(z_{i}^{s}-z_{i}^{r}\right)-\sum_{i=1}^{p}\left(z_{0 i}^{s}-z_{0 i}^{r}\right)-\sum_{i=1}^{p}\left(s z_{0 i}^{s-1}-r z_{0 i}^{r-1}\right)\left(z_{i}-z_{0 i}\right)\right]
$$

no caso em que $s \rightarrow 1$ e $r>0$

$$
D_{1, r}\left(z, z_{0}\right)=\frac{1}{1-r}\left[\sum_{i=1}^{p}\left(z_{i}-z_{i}^{r}\right)-\sum_{i=1}^{p}\left(z_{0 i}-z_{0 i}^{r}\right)-\sum_{i=1}^{p}\left(1-r z_{0 i}^{r-1}\right)\left(z_{i}-z_{0 i}\right)\right]
$$

e no caso em que $s \rightarrow 1$ e $r \rightarrow 1$

$$
D_{1,1}\left(z, z_{0}\right)=-\sum_{i=1}^{p}\left(z_{i}-z_{0 i}-z_{i} \ln \frac{z_{i}}{z_{o i}}\right)
$$




\section{O Algoritmo ART}

Enumere-se todos os cones que particionam o domínio com o índice $k, k=1, \ldots K$, onde $K$ é o número total de cones. A relação entre $k, j$ número de vista, e $n_{j}$ número de cones é dada por $k=\left(j N_{j}-1\right)+n_{j}$. Considere-se a matriz $A$, com dimensões $(K, E)$ onde cada linha de $A$ esta relacionado a um cone e uma coluna de $A$ a um elemento de área da partição do domínio. A linha $k$ contém todas as informações dos elementos de área que formam o cone $k$. A Eq. (1.3) pode ser escrita como

$$
h(k, 1)=\int_{\text {cone }_{j, n_{j}}} f(x, y) d s, \quad h=A \sigma,
$$

onde $\sigma$ é $h$ são vetores coluna. Define-se a função erro como a diferença entre a medida experimental do detector e o calculado.

$$
F=h-A \sigma
$$

e a Lagrangiana

$$
L_{1, r}\left(\sigma, \sigma_{0}, \lambda\right)=D_{1, r}\left(\sigma, \sigma_{0}\right)+\lambda^{T} F,
$$

onde $\lambda$ é um vetor coluna formado pelos multiplicadores de lagrange. Sendo $E>K$ o problema inverso é solucionado como um problema de otimização, e deve cumprir com dois requerimentos:

1.-

$$
\nabla_{\sigma} L=\nabla_{\sigma} D_{1, r}+\nabla_{\sigma}\left(\lambda^{T} F\right)=0
$$

onde

$$
\nabla_{\sigma}\left(\lambda^{T} F\right)=A^{T} \lambda
$$

para $r=1$ ou $r \neq 1$ respectivamente temos

$$
\nabla_{\sigma} D_{1,1}=\ln \sigma-\ln \sigma_{0}, \quad \nabla_{\sigma} D_{1, r}=\frac{r}{r-1}\left[\sigma^{r-1}-\sigma_{0}^{r-1}\right]
$$

das Eqs. (4.4),(4.5) e (4.6) obtemos para $r=1$ ou $r \neq 1$

$$
\ln \sigma=\ln \sigma_{0}-A^{T} \lambda, \quad \sigma^{r-1}=\sigma_{0}^{r-1}-\frac{r-1}{r} A^{T} \lambda,
$$

cada elemento $e$ de $\ln \sigma$ ou de $\sigma^{r-1}$ respectivamente tem a forma $\ln \sigma(e)$ ou $(\sigma(e))^{r-1}$, conseqüentemente os elementos de $\sigma$ podem ser calculados sem dificuldade.

2.-

$$
\nabla_{\lambda} L=\nabla_{\lambda} D_{1, r}+F+\left(\nabla_{\lambda} F\right) \lambda=0
$$

conseqüentemente, tem-se

$$
\lambda=-\left(\nabla_{\lambda} F\right)^{-1}\left(F-\nabla_{\lambda} D_{1, r}\right),
$$

onde

$$
\begin{gathered}
\nabla_{\lambda} D_{1, r}=\nabla_{\lambda} \sigma \nabla_{\sigma} D_{1, r} \\
\nabla_{\lambda} F=\nabla_{\lambda} \sigma A^{T} .
\end{gathered}
$$


Se $r=1$ temos

$$
\left[\nabla_{\lambda} \sigma\right](k, e)=\frac{\partial \sigma_{e}}{\partial \lambda_{k}}=-\sigma_{e} A(k, e)
$$

caso $r \neq 1$

$$
\left[\nabla_{\lambda} \sigma\right](k, e)=-\frac{1}{r} \sigma_{e}^{2-r} A(k, e) .
$$

O problema é resolvido em forma iterativa, com o seguinte algoritmo:

Para $u=1,2 \ldots$ ate que a convergencia seja obtida

Se $u=1$, dado um $\sigma_{0}$, empregue as Eqs.(4.2, 4.11 e 4.12)

e calcule $\lambda=-\left(\nabla_{\lambda} F\right)^{-1} F$

Se $r=1: \ln \sigma_{1}=\ln \sigma_{0}-A^{T} \lambda$

Caso contrario : $\sigma_{1}^{r-1}=\sigma_{0}^{r-1}-\frac{r-1}{r} A^{T} \lambda$

Se $u>1$ empregue as Eqs.(4.2, 4.11 e 4,12) e calcule a Eq, (4.5)

$\nabla_{\sigma} D_{1,1}=\ln \sigma_{u-1}-\ln \sigma_{u-2}$, ou $\nabla_{\sigma} D_{1, r}=\frac{r}{r-1}\left(\sigma_{u-1}^{r-1}-\sigma_{u-2}^{r-1}\right)$

calcule $\lambda=-\left(\nabla_{\lambda} F\right)^{-1}\left(F-\nabla_{\lambda} D_{1, r}\right)$

logo calcule respectivamente caso seja $r=1$ ou $r \neq 1$

$\ln \sigma_{u}=\ln \sigma_{u-1}-A^{T} \lambda, \quad \sigma_{u}^{r-1}=\sigma_{u-1}^{r-1}-\frac{r-1}{r} A^{T} \lambda$

Se $\sum_{e=1}^{E}\left|\sigma_{u}(e)-\sigma_{u-1}(e)\right| /\left|\sigma_{u}(e)\right|<\varepsilon$, fim.

\section{Resultados}

Procurando um número que nos indique a qualidade nas estimativas das incógnitas em cada caso teste, definimos o erro quadrático relativo médio $(E P)$, dado por:

$$
E P=\frac{100 \sqrt{\sum_{e=1}^{E}\left(\frac{\sigma_{e, e x a t o}-\sigma_{e, \text { calculado }}}{\sigma_{e, \text { exato }}}\right)^{2} a_{e}^{2}}}{a_{V}},
$$

onde $\sigma_{e}$ é o coeficiente de absorção do elemento de área $e, E$ o número total de elementos e $a_{V}$ a área do domínio $V$.

Neste trabalho, para os casos testes, foram empregados como medidas experimentais dados gerados com ruído pseudo-randômicos obtido por rotinas computacionais,

$$
q_{i, \text { experimental }}=q_{i, \text { exato }}+\nu_{i} \chi,
$$

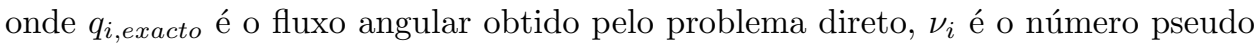
randômico com distribuição uniforme e $\chi$ simula o nível de ruído, com diferentes valores de $\chi$ podem ser simulados diversos níveis de ruído. Os números randômicos são gerados com a função RAND do MATLAB.

Partição de domínio. Na Figura 2, apresenta-se o domínio empregado, com Ro $=50 \mathrm{~cm}$, para meios heterogêneos as perturbações são círculos com $\mathrm{R}=10 \mathrm{~cm}$ e $\mathrm{R}^{\prime}=8 \mathrm{~cm}$. O coeficiente de atenuação no meio, é $\sigma_{a}$ e nas perturbações $\sigma_{a 1}=$ $10 \sigma_{a}$ e $\sigma_{a 2}=5 \sigma_{a}$ todos em $\mathrm{cm}^{-1}$. O valor inicial para os coeficiente de atenuação em todos os casos testes foi $\sigma_{0}=3$.

O número total de elementos de área que resulta da discretização do domínio, esta relacionada com o número de cones e de rotações (vistas) que forma o sistema. A Figura 3 apresenta a relação entre o número de cones, vistas e elementos de área 


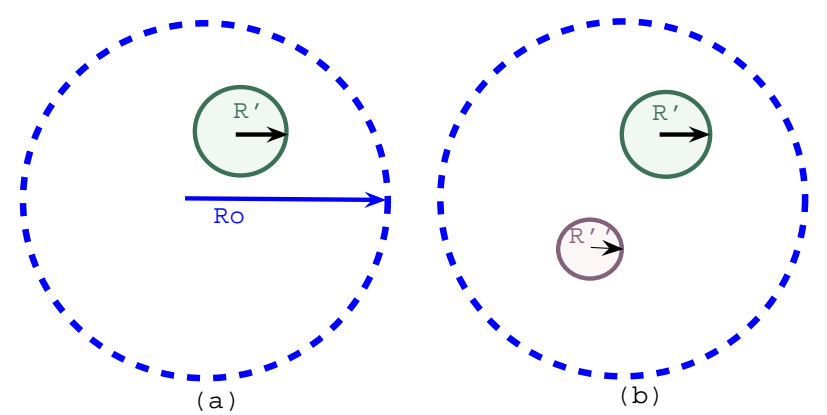

Figura 2: Configurações usadas nos casos testes. (a) Configuração 1: meio com uma deformação. (b) Configuração 2: meio com duas deformações.

gerado pela discretização. Os sistemas a serem resolvidos estão formados por $E$ incógnitas e $J N_{j}$ dados. Em uma partição de 3 vistas e 80 cones tem-se 11172 incógnitas e 240 dados.

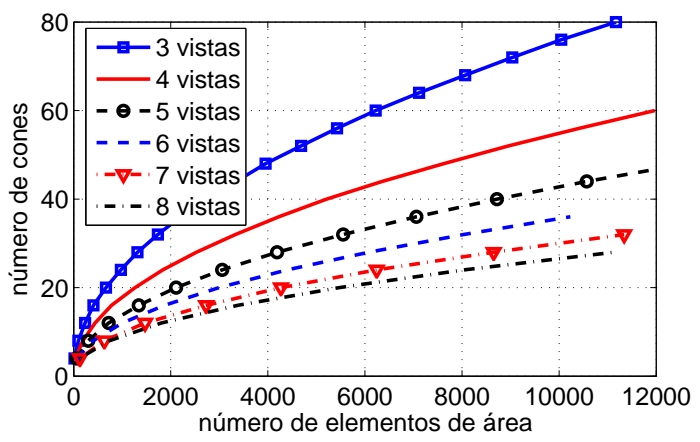

Figura 3: Número de elementos de área versus o número de cones e vistas

Caso Teste 1: Meio Homogêneo. Considerando, um meio homogêneo com $\sigma_{a}=0,2$, valor inicial $\sigma_{0}=3$, dados com ruído pseudo - randômico até $1 \%$ e empregando o funcional $\eta_{1, r} \operatorname{com} r=1$. As Figuras 4 apresentam o erro quadrático relativo médio $(E P)$ da solução do problema inverso calculado para diferentes números de elementos de área em que está particionado o domínio. Observa-se que a maior discretização o $E P$ diminui. O menor $E P$ é obtido com a discretização de 3 vistas e 80 cones. O algoritmo converge em 2 iterações para $\varepsilon=0.0001$.

Caso Teste 2: Meio Heterogêneo. A melhor discretização. Considerando, as configurações 1 e 2 apresentadas na Figura $2, \sigma_{a}=0,1, \sigma_{a 1}=10 \sigma_{a}, \sigma_{a 2}=5 \sigma_{a}$, e $\sigma_{0}=3,0$, dados com ruído pseudo randômico ate $1 \%$, para solução do problema inverso emprega-se o funcional $\eta_{1, r} \operatorname{com} r=1$. As Figuras 5 apresentam o erro $E P$ versus o número de elementos de área gerados para um meio com uma e duas perturbações respectivamente. Observa-se que o $E P$ descresse com o incremento do 


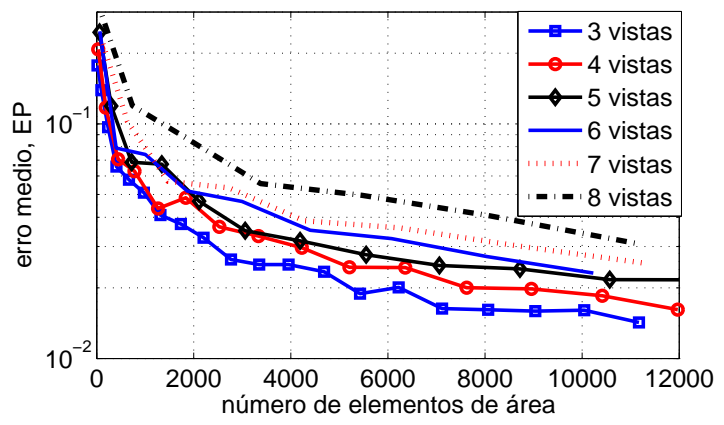

Figura 4: Erro médio versus número de elementos de área e vistas

número de elementos de área no domínio. O menor EP é obtidos com 3 e 5 vistas. Para meios heterogêneos considerou-se $\varepsilon=0,0005$.
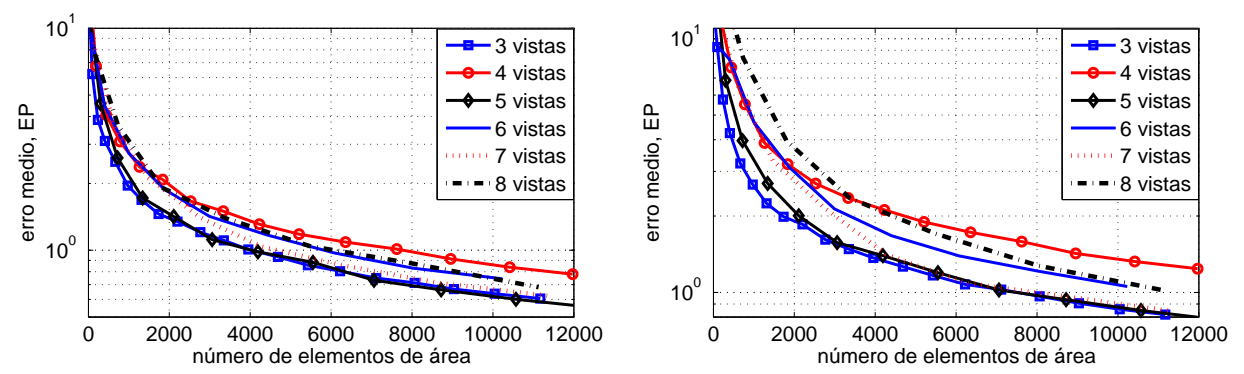

Figura 5: Erro médio versus número de elementos de área. Meio com 1 e 2 perturbações respectivamente.

Caso Teste 3: Meio Heterogêneo. O melhor $r$. Considerando; as configurações 1 e 2 apresentadas na Figura 2, as partições que geram o maior número de elementos de áreas, $\sigma_{a}=0,1, \sigma_{a 1}=10 \sigma_{a}, \sigma_{a 2}=5 \sigma_{a}, \sigma_{0}=3,0$, e dados com ruído ate 1\%. Nas Figuras 6 apresentam-se os $E P$ variando $r$ no intervalo [0,1-2] para um meio com uma e duas perturbações respectivamente. Observa-se que o menor $E P$ é obtido para $r=0,1$ e em um meio particionado por 3 e 5 vistas. Nas Figuras 7 para as configurações 1 e 2 respectivamente apresentam-se os $E P$ variando $r$ no intervalo [0,1-2], para $\sigma_{a}=0,1,0,2,0,4,0,6,0,8,1, \sigma_{a 1}=10 \sigma_{a}, \sigma_{a 2}=5 \sigma_{a}, \sigma_{0}=$ 3,0 , dados com ruído randômico ate $1 \%$ e uma partição de domínio com 3 vistas, o menor $E P$ também é obtido para $r=0,1$. Observa-se que os $E P$ não variam com o coeficiente de atenuação a estimar, os $E P$ variam para diferentes valores de $r$ e com a percentagem de ruído. O algoritmo converge em 5 iterações.

Caso Teste 4: Reconstrução com Ruído. Considerando as configuração 1 da Figura 2, $\sigma_{a}=1,0, \sigma_{a 1}=10, \sigma_{0}=3,0$ e dados com ruído randômico até 5 $\%$. A Figura 8 apresentam a imagem original e a reconstruída da configuração 1 empregando $r=0,1$, e uma partição de 3 vistas e 80 cones. A Figura 9 apresentam 

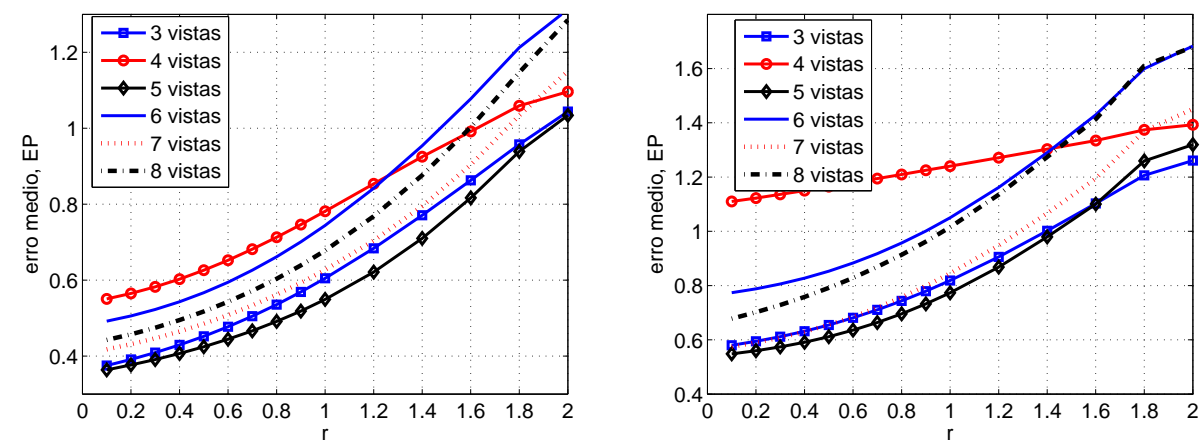

Figura 6: EP versus $r$. Meio com 1 e 2 perturbações respectivamente.
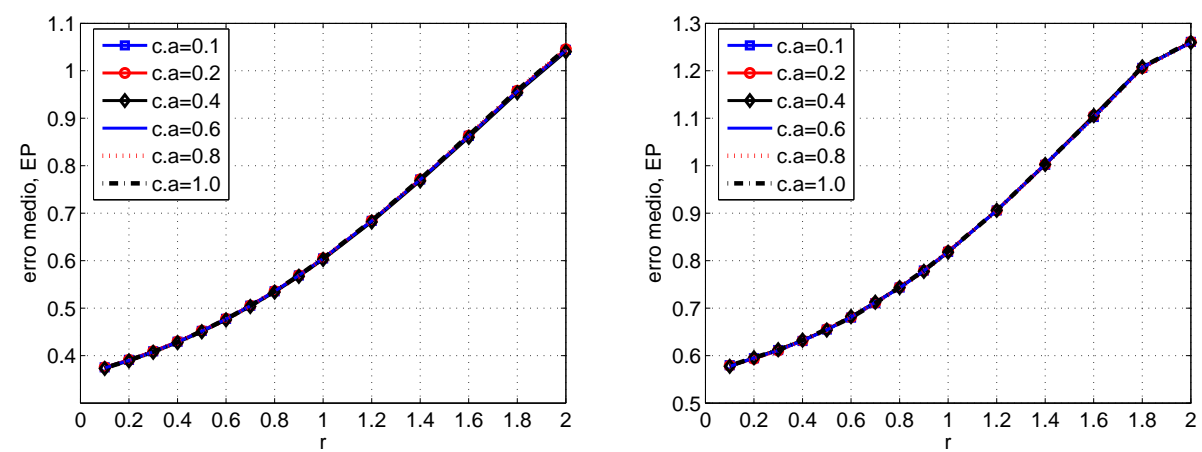

Figura 7: EP versus $r$. Meio com 1 e 2 perturbações respectivamente

a imagem original e a reconstruída para a configuração 2 , considerando $5 \%$ de ruído randômico com $\sigma_{a}=0,1, \sigma_{a 2}=1,0$, e $\sigma_{0}=3,0, r=0,1$ e a discretização de domínio é de 3 vistas e 80 cones.

\section{Conclusões}

Neste trabalho empregamos o funcional $\eta_{1, r}$ no algoritmos ART para a reconstrução de imagens, os parâmetros $r=0,1$ e $s \rightarrow 1$ ofereceram os melhores resultados na solução do problema inverso, quando os dados apresentam ruído randômico, superando a função relacionada à entropia de Shannon para $r=1$ e $s \rightarrow 1$. A melhor partição de domínio é onde obteve-se a melhor estimativa dos coeficientes de atenuação que foi com 3 e 5 vistas. Em todos os casos heterogêneos o algoritmo converge em 5 iterações. Em futuros trabalhos usaremos dados gerados com o método de Monte Carlo. 

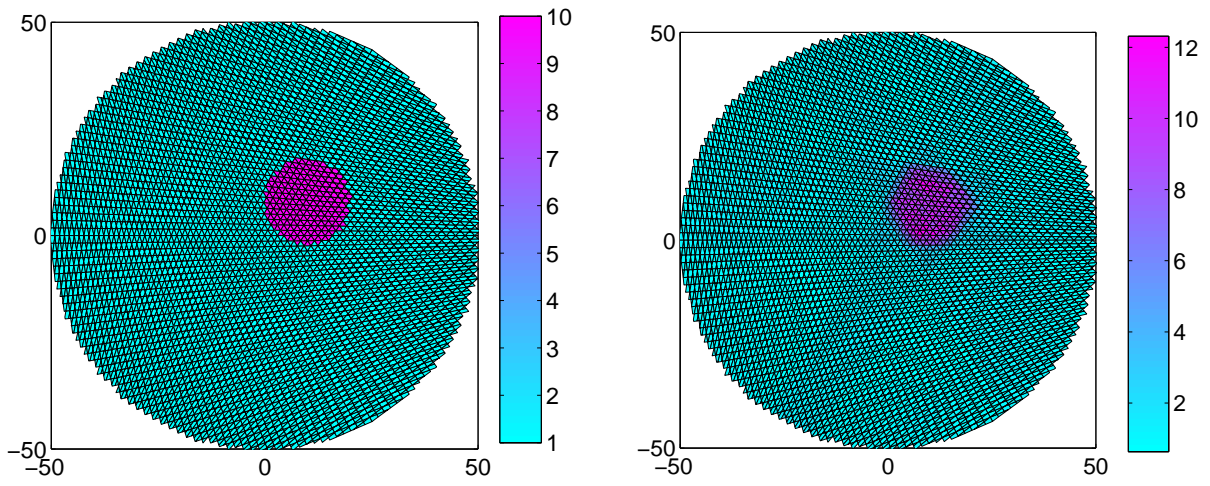

Figura 8: Reconstrução com 3 vistas. Para a configuração 1. $E P=0.3715$.
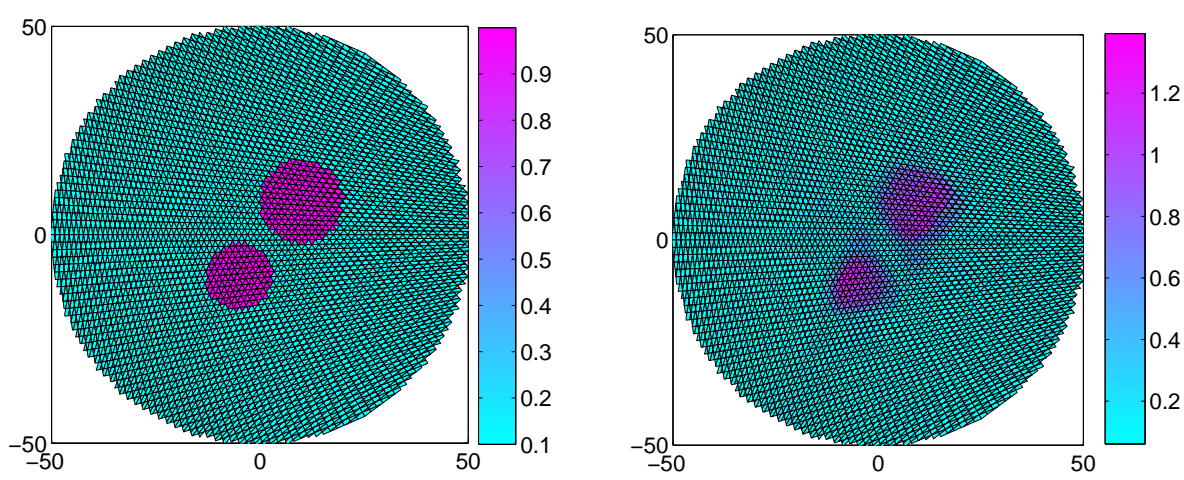

Figura 9: Reconstrução com 3 vistas. Para a configuração 2. $E P=0.5877$.

\section{Agradecimentos}

Os autores agradecem ao Prof. J.P. Zubelli pelo apoio e sugestões para o desenvolvimento deste trabalho. R.F. Carita Montero agradece à CNPq (Conselho Nacional de Desenvolvimento Científico e Tecnológico) pelo suporte financeiro, com o número 30.0024 .

Abstract. We consider a class of Algebraic Reconstruction Technique (ART) algorithms for the absorption coefficient using certain Bregman functions. The Bregman functional is related to a class of entropies studied by Shannon, Harvda-Charvát and Sharma - Taneja. This functional depends on certain parameters $\mathrm{r}$ and s and is strictly convex. Using it we build a family of Bregman distances to be used in the ART algorithms for image reconstruction with divergent beams. In this work we seek, the optimal values for the parameter $r$ when $s \rightarrow 1$, the initial guess values and an appropriate discretization of the medium that offers the best results for the absorption coefficient identification. 


\section{Referências}

[1] L.M. Bregman, The relaxation method of finding the common point of convex sets and its application to the solution of problems in convex programming, USSR Computational and Mathematical Physics Journal, 7 (1967), 200-217.

[2] R.F. Carita Montero, N.C. Roberty, A.J. Silva Neto, Absorption coefficient estimation in heterogeneous media using a domain partition consistent with divergent beams, Inverse Problems in Engineering, 9 (2001), 587-617.

[3] D. Dicken, A new approach towards simultaneous activity and attenuation reconstruction in emission tomography, Inverse Problems, 15 (1999), 931-960.

[4] J. Havdra, F. Charvát, Concept of Structural $\alpha$-Entropy, Kybernetika, 3 (1967), 30-35.

[5] A.C. Kak, M. Slaney, "Principles of Computerized Tomographic Imaging", IEEE Press, 1988.

[6] W.A. Kalender, X-Ray computed tomography - State of the art, em "Medical Optical Tomography: Functional Imaging and Monitoring" (R.F. Potter ed.), pp. 11-27, IS11 SPIE Bellingham, Washington, 1993.

[7] M.L. Reis, N.C. Roberty, Maximum entropy algorithms for image reconstruction from projections, Inverse Problems, 8 (1992), 623-644.

[8] C.E. Shannon, A mathematical theory of communication. Bell, System Tech. J., 27 (1948), 379-423.

[9] B.D. Sharma, I.J. Taneja, Entropy of type $(\alpha, \beta)$ and other generalized measures in information theory, Metrika, 22 (1975), 205-215. 